\title{
ALGUNAS IMPLICACIONES TERRITORIALES DE LA INTERVENCIÓN ADMINISTRATIVA EN LA GESTIÓN DEL PARQUE NATURAL DE LA SIERRA DE CARDEÑA Y MONTORO (CÓRDOBA)*
}

\author{
Garzón García, R. \\ Departamento de Geografía y Ciencias del Territorio. Universidad de Córdoba.
}

\begin{abstract}
Resumen: Son pocos los trabajos que desde la Geografía han profundizado en el estudio de las líneas de actuación implementadas en los espacios naturales protegidos así como de las repercusiones territoriales de las mismas. En esa línea, el presente estudio plantea un primer acercamiento a la cuestión, tomando como referencia el Parque Natural de la Sierra de Cardeña y Montoro (Córdoba).
\end{abstract}

En base a una serie de factores que se analizan en el texto, en este espacio se ha venido desarrollando una gestión en la que han primado las labores de conservación, de mejora forestal y control de las actividades agrarias tradicionales; por el contrario, ha sido mucho menor el desarrollo experimentado por otras líneas de acción, también consustanciales a la figura de Parque Natural andaluz -como el fomento del uso público y el desarrollo endógeno-, las cuales, sin embargo, parecen comenzar a ser objeto de un mayor impulso administrativo en la actualidad.

Palabras clave: Sierra Morena, Parque Natural, Administración, gestión, territorio.

\begin{abstract}
There are not many geographical works about the study of actions developed on natural protected areas and their results on territory. Therefore, this paper offers an approach to this question, referring to the Sierra de Cardeña y Montoro Natural Park (Córdoba).
\end{abstract}

Due to several factors which are analysed in the text, the predominance of conservation, forest improvement and control of traditional agricultural exploitation have characterized the management developed in this area up to now. On the contrary, other kinds of actions also linked to andalusian Natural Park -like the public use and the development of this area- start to be more stimulated at the moment.

Key words: Sierra Morena, Natural Park, Administration, management, territory.

* Recibido: 10-11-03. Aceptado: 4-7-04. 


\section{Introducción}

Tal como se ponía de manifiesto en un trabajo publicado una década atrás (Gómez Mendoza et alii, 1994), son pocos -aún en la actualidad- los trabajos que reparan en la cuestión de los espacios naturales protegidos desde la óptica de la gestión, es decir, de las líneas de actuación desarrolladas en los mismos y de las repercusiones que sobre el territorio tienen dichas actuaciones.

En este sentido, consideramos que nos encontramos ante un importante déficit en el estudio geográfico de dichas áreas, por cuanto que los espacios naturales protegidos se configuran en la actualidad, a nuestro entender, como uno de los principales factores de transformación territorial de extensas zonas; y ello no sólo por la gran expansión que han experimentado desde los años ochenta en nuestro país (Mulero Mendigorri, 2002), sino, sobre todo, por los ambiciosos objetivos planteados para muchos territorios que son declarados como tales (EuroparcEspaña, 2002). Uno de los casos paradigmáticos a este respecto es el referido a la figura de Parque Natural andaluz, concebido desde su origen ${ }^{1}$ como un instrumento que debe contribuir a fomentar tanto la conservación, como el desarrollo endógeno y el uso público recreativo de los espacios en que fuese de aplicación (Azcárate y Bang, 1986).

Se propone, pues, en este trabajo, el análisis y valoración de las líneas de actuación más destacadas emprendidas en un Parque Natural andaluz (el de la Sierra de Cardeña y Montoro), desde el momento de su declaración (año 1989) hasta el presente, así como de las principales implicaciones que las mismas han tenido sobre el territorio protegido. Dicho análisis, además de contribuir a sintetizar las claves del manejo de este espacio, nos permitirá igualmente realizar algunas reflexiones de carácter más genérico acerca de los mecanismos y algunas de las principales tendencias que han marcado hasta la actualidad la gestión de los Parques Naturales andaluces.

Uno de los factores que quizá contribuya más decididamente a la escasez de trabajos en esta línea es la frecuente carencia de fuentes que proporcionen una información detallada, fiable y prolongada en el tiempo. Para el Parque Natural de la Sierra de Cardeña y Montoro hemos podido contar con un elenco de fuentes bastante completo: instrumentos básicos de planificación (PORN y PRUG), actas de todas las sesiones celebradas por la Junta Rectora del Parque Natural, documentación diversa proporcionada desde la Delegación Provincial de la Consejería de Medio Ambiente en Córdoba ${ }^{2}$ y, sobre todo, las Memorias de Actividades del Parque Natural. 


\section{Principales factores explicativos de la gestión desarrollada en el Parque Natural de la Sierra de Cardeña y Montoro}

\subsection{La base territorial del espacio protegido}

En el caso del Parque Natural de la Sierra de Cardeña y Montoro se trata, con sus 38.449 hectáreas $^{3}$, de un parque de tamaño medio, tanto en el contexto andaluz como en el de Sierra Morena. Se encuentra situado en el sector más nororiental de la Sierra Morena cordobesa (figura 1), limitando al oeste con el sector central del espacio mariánico cordobés y el batolito granítico de los Pedroches (del que participa en su mitad más septentrional), al sur con los amplios espacios de olivar del término municipal de Montoro (que se prolongan hasta el mismo Valle del Guadalquivir), al este con el Parque Natural de la Sierra de Andujar (del que se encuentra separado por el Río Yeguas), y al norte con la provincia de Ciudad Real.

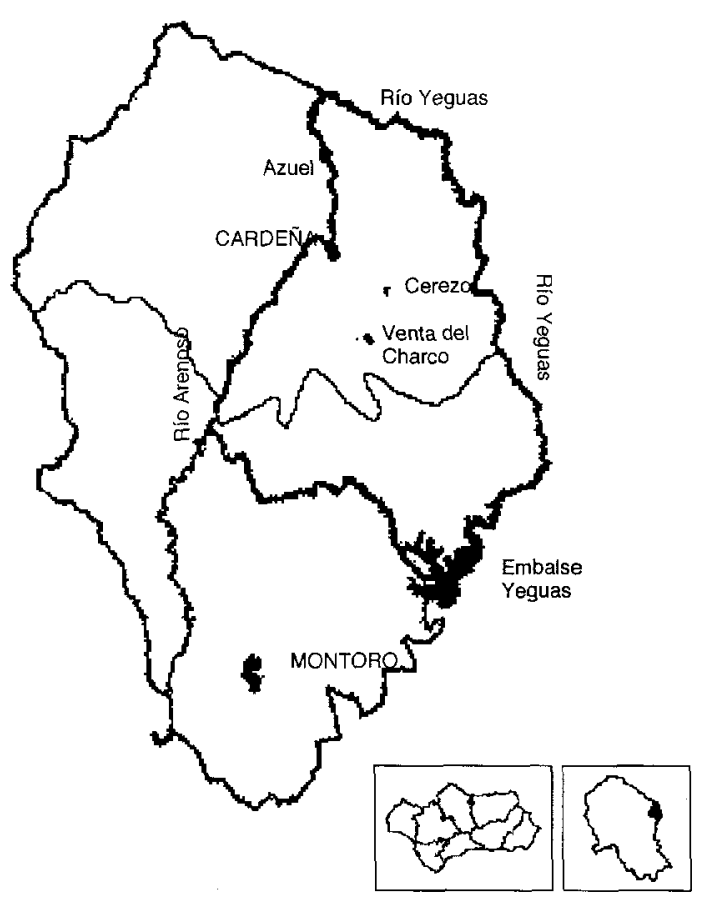

Figura 1. Mapa de localización del Parque Natural en el contexto de los términos municipales de Cardeña y Montoro.

Fuente: Elaboración propia a partir del Mapa Digital de Andalucía (1997). 
Desde un punto de vista orográfico se pueden distinguir en este Parque Natural tres zonas claramente diferenciadas: el sector más meridional (coincidente a grandes rasgos con lo que es el término municipal de Montoro dentro del ámbito protegido) de topografia abrupta, intrincada y acusadas pendientes por lo general, perteneciente a la franja de relieve más estrictamente montano que recorre en sentido longitudinal la Sierra Morena cordobesa; un relieve igualmente quebrado se manifiesta en los extremos oriental y occidental del perímetro protegido, coincidentes con los profundos valles fluviales de los ríos Yeguas y Arenosos, los dos principales cursos que drenan este espacio; finalmente, la zona norte y central pertenece litológicamente al extenso batolito granítico que abarca gran parte del norte de la provincia de Córdoba, presentando por ello una topografía mucho más suave, a modo de penillanura elevada.

El ámbito protegido incluye una notable variedad de unidades territoriales y paisajísticas que, en todo caso, pueden sintetizarse en cuatro unidades básicas: zonas de dehesa de quercíneas (emplazadas básicamente en la zona central y septentrional, de formas suaves y aplanadas), en las que la encina resulta la especie absolutamente mayoritaria si bien se localizan algunos elementos especialmente destacables, tales como algunas formaciones de roble melojo; espacios de bosque mediterráneo autóctono (coincidentes con las áreas de topografía más quebrada), que gozan en no pocos casos de un gran estado de conservación, sirviendo como hábitat a algunas especies de fauna de gran vulnerabilidad como el lobo o el lince ibérico; pequeños enclaves de olivar marginal, situados en una pequeña porción de terreno de la zona suroriental del Parque; y, finalmente, las zonas de repoblación forestal de coníferas -con dos especies predominantes, Pinus pinea y Pinus pinaster-, localizadas tanto en el entorno de las cuencas fluviales de los ríos Arenoso y Yeguas como en la franja más meridional del Parque Natural, constituyendo una serie de teselas de bosques de pinar (figura 2).

El elevado grado de conservación general de los ecosistemas del Parque Natural se debe en gran medida al hecho de tratarse éste, tradicionalmente, de un área marginal desde el punto de vista poblacional. De hecho, la población dentro del perímetro protegido se limita al núcleo de Venta del Charco (Cardeña), con un censo actual aproximado de 188 habitantes (Consejería de Medio Ambiente, 2001b) ${ }^{4}$, así como a la escasa población residente -generalmente de forma temporal- en las edificaciones existentes en las explotaciones agrarias del Parque Natural. El núcleo de Montoro 5 se encuentra, por su parte, bastante alejado de los límites del espacio protegido, y la cercanía de los núcleos de Cardeña y su aldea Azuel (figura 1) se ve en buena medida atenuada ante el escaso peso demográfico de ambos ${ }^{6}$.

Dicha debilidad demográfica se explica, sobre todo, a tenor de dos factores fundamentales: primeramente, la escasa potencialidad productiva de los suelos para las labores agrícolas, que ha condicionado en gran medida los aprovechamientos a des- 


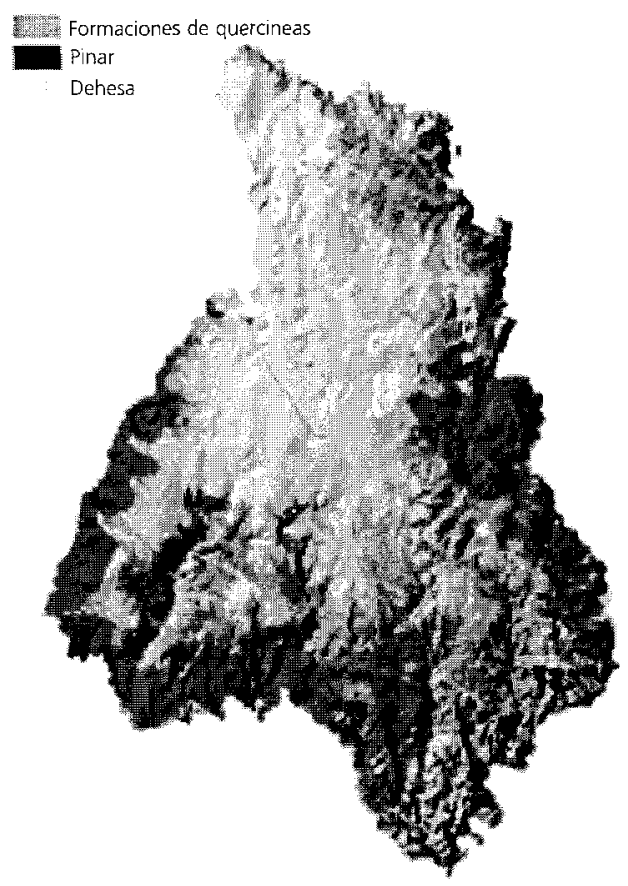

Figura 2. Distribución relativa de las principales formaciones vegetales del Parque Natural de la Sierra de Cárdeña y Montoro.

Fuente: Consejería de Medio Ambiente (2001b).

arrollar en este territorio; y, en segundo lugar, la peculiar estructura de la propiedad, patente en este espacio. Ésta se caracteriza por un absoluto predominio de los predios privados (cuadro 1), destacando en ese contexto general un número relativamente reducido de grandes fincas -de extensión superior a las 500 hectáreas- que abarcan por sí solas en torno a un 85-90\% del territorio en régimen de titularidad privada. Esta gran propiedad privada -coincidente, muy mayoritariamente, con grandes cotos de caza mayor-, se localiza, sobre todo, en los amplios espacios adehesados y zonas de bosque de quercíneas en los terrenos más quebrados del Parque Natural?

Por su parte, existe, en el marco de esta desequilibrada estructura de la propiedad, un amplio número de pequeños predios que no representan, sin embargo, más que una reducida porción superficial del territorio protegido. Éstos aparecen localizados fundamentalmente, en un triángulo emplazado entre el núcleo de Cardeña y las aldeas de Azuel y El Cerezo (coincidentes con espacios de dehesa, entre los que se intercalan algunos huertos vinculados a pequeñas explotaciones familiares), así como en los enclaves de olivar marginal al sureste del área protegida. 
Cuadro 1. Distribución según tipos de propiedad de la tierra en el Parque Natural de la Sierra de Cardeña y Montoro.

\begin{tabular}{|lcc|}
\hline Tipo de propiedad & Superficie (has) & $\%$ \\
\hline Junta de Andalucia & $526 \% 37$ & $1 \% 37$ \\
Municipal & $4 \% 12$ & $0^{\prime} 01$ \\
Particulares & $29.970^{\prime} 19$ & $77^{\prime} 96$ \\
Particulares consorciados o convenidos & $7.948^{\prime} 32$ & $20^{\prime} 66$ \\
Total & 38.449 & 100 \\
\hline
\end{tabular}

Fuente: Elaboración propia a partir del Sistema de Información para la Gestión de los Montes y sus Aprovechamientos (SIGMA) (Consejería de Medio Ambiente de la Junta de Andalucía), e información interna facilitada por la Delegación de dicha Consejería en Córdoba.

Junto a todo ello, en el cuadro 1 puede detectarse otra realidad aún más específica de este espacio protegido, como es la considerable presencia de predios (incluidos generalmente entre las grandes propiedades del Parque Natural) consorciados y convenidos para su repoblación forestal. Tal como tendremos ocasión de exponer en el próximo apartado de este trabajo, estas fincas, en función de sus especiales condiciones en el momento presente, absorben buena parte de los esfuerzos de la Administración ambiental andaluza en la gestión de este Parque Natural.

En cuanto a los aprovechamientos, destaca en primer lugar la explotación ganadera tradicional en la zona, vinculada fundamentalmente a los espacios de dehesa, la cual presenta un carácter eminentemente extensivo, más acusado en las grandes fincas privadas. Las cabañas más numerosas son la ovina y la porcina, seguidas por la bovina y ya, en un plano muy secundario, por otras como la caprina y la equina (Consejería de Medio Ambiente, 2001b). Una realidad ajena en gran medida a las explotaciones de pequeño y mediano tamaño, pero cada vez más patente en las grandes propiedades la constituye la compatibilidad de la explotación cinegética y ganadera, esencialmente en aquellas superficies adehesadas que permiten el aprovechamiento del pasto tanto por parte del ganado doméstico como de las reses cinegéticas. A este respecto, es necesario resaltar asimismo el crecimiento sostenido durante los últimos años de la actividad cinegética, vinculada esencialmente a los cotos de caza mayor ${ }^{8}$. Junto a éstos, hay que hacer referencia a otros aprovechamientos que, si bien no gozan del peso específico de los anteriores, contribuyen sin duda a caracterizar este espacio como de vocación muy mayoritariamente agraria: los forestales (el ramoneo, la leña, y el corcho en las superficies adehesadas, o la explotación maderera en masas de pinar) y los agrícolas (siembras -sobre todo de gramíneas y leguminosas, para aprovechamiento a diente por el ganado-, así como los cultivos leñosos de olivar, de escasa relevancia tanto superficial como productiva). 


\subsection{Medios específicos para la gestión del Parque Natural}

Este espacio fue objeto, algunos años después de su declaración, de una ordenación de sus usos y actividades, a raíz de la aprobación, mediante Decreto 121/1994, de 31 de mayo, de su Plan de Ordenación de los Recursos Naturales (PORN) y su Plan Rector de Uso y Gestión (PRUG). Dichos documentos han sido revisados y actualizados en fechas recientes (Consejería de Medio Ambiente, 2003), en cumplimiento de los plazos legales de vigencia y a tenor de las nuevas circunstancias que caracterizan a este Parque Natural en la actualidad. No obstante, las referencias ofrecidas en este trabajo aluden a los documentos originarios aparecidos en 1994, por cuanto han sido aquéllos los que han estado en vigor durante el lapso de tiempo objeto de análisis a través de esas líneas.

Cabe señalar que ambos documentos de referencia para la gestión se concibieron básicamente como instrumentos normativos, estableciéndose en el PORN la regulación específica de usos y actividades, mientras que en el segundo se contemplan las condiciones y requisitos técnicos para el desarrollo de los mismos así como las normas para la gestión y administración del Parque Natural.

La orientación que inspiró la elaboración de sendos instrumentos de planificación adoleció, con carácter general, de un marcado matiz protector, reflejado con gran nitidez a través del listado de objetivos recogido en el artículo 4 del PORN (Consejería de Medio Ambiente, 1996b), en el que resultaban claramente predominantes aquellos orientados a la conservación y mejora del patrimonio natural del Parque. No obstante, en el mismo PORN se diseñó una zonificación del espacio (figura 3), acompañada de la definición de una normativa particular con distintos niveles de protección para cada uno de las áreas diferenciadas en la misma. Se distinguen tres niveles (A, B, y C) según la mayor o menor relevancia ecológica de los enclaves incluidos en cada uno de ellos.

- Así, en las Zonas de Protección Grado A se incluyen: los montes públicos del Parque Natural (calificadas como Áreas de Alto Interés Ecológico), los principales cauces fluviales (bajo la denominación de Complejos Fhuviales: valles del río Yeguas, arroyo Arenoso y arroyo de los Frailes), así como los robledales existentes en la zona central de este espacio (Áreas de Vegetación Especial).

- Las Zonas B comprenden los espacios de dehesa y formaciones de quercíneas (bosque autóctono y áreas de matorral), las repoblaciones de coniferas, los olivares $^{9}$, así como el Embalse del Yeguas.

- Zonas de Protección de Grado C, limitadas a la aldea de Venta del Charco y las viviendas rurales que conforman el núcleo de El Cerezo (Consejería de Medio Ambiente, 1996b, 62-63). 


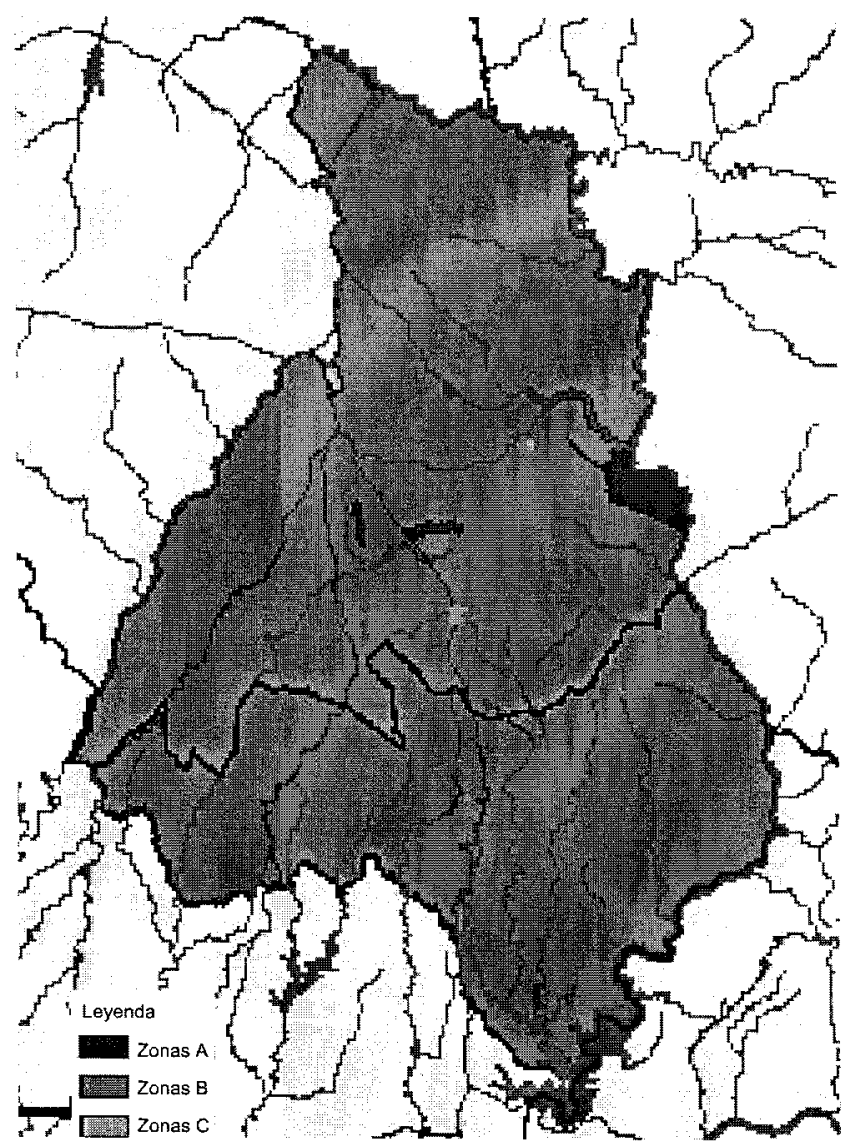

Figura 3. Zonificación del Parque Natural de la Sierra de Cardeña y Montoro según el PORN de 1994. Fuente: www.juntadeandalucia.es/medioambiente Elaboración propia.

Tanto el PORN como el PRUG mostraban, a nivel de contenidos, un excesivo carácter regulador o normativo, resultando por el contrario inexistente cualquier tipo de propuesta de actuación a emprender en este espacio protegido ${ }^{10}$.

A esta última carencia aludida se añade una segunda realidad que condiciona en gran medida la gestión a desarrollar: la relativa a la inexistencia en este Parque Natural, al igual que en el resto de Parques andaluces, de un presupuesto específico e individualizado para el mismo.

Ello no significa, no obstante, que no se hayan ejecutado inversiones en este espacio protegido (cuadro 2), si bien ellas proceden de los presupuestos generales de la 
Cuadro 2. Inversiones -según conceptos- de la Administración ambiental andaluza en el Parque Natural de la Sierra de Cardeña y Montoro (1990-2002).

\begin{tabular}{|lcc|}
\hline Conceptos & Gastos (en ptas/euros) & $\%$ \\
\hline Tratamientos selvícolas & $655.116 .365 / 3.930 .698^{\prime} 1$ & $56^{\prime} 51$ \\
Prevención de incendios y lucha contra plagas* & $103.336 .956 / 620.021^{\prime} 7$ & $8^{\prime} 91$ \\
Protección y mejora de flora y fauna amenazada & $33.655 .043 / 201.930^{\prime} 25$ & $2^{\prime} 90$ \\
Estudios e investigación & $10.750 .000 / 64.500$ & $0^{\prime} 93$ \\
Obras e infraestructuras & $185.594 .061 / 1.113 .564^{\prime} 3$ & $16^{\prime} 01$ \\
Uso público & $133.436 .687 / 800.620^{\prime} 14$ & $11^{\prime} 51$ \\
Otros & $37.401 .015 / 224.4066^{\prime} 09$ & $3^{\prime} 23$ \\
\hline
\end{tabular}

Fuente: Elaboración propia a partir de las Memorias Anuales de Actividades del Parque Natural.

* No se contemplan los datos de 1998, 1999, 2000 y 2002 de tratamientos preventivos, y de 1991,

1993, 1994 y 1994 de lucha contra plagas, al no encontrarse disponibles al elaborar este trabajo.

Consejería de Medio Ambiente de la Junta de Andalucía ${ }^{11}$, la cual establece unos programas prioritarios de actuación para cada Parque Natural que son financiados con cargo a dichos presupuestos.

De la observación del cuadro 2 se deduce con nitidez que en las acciones emprendidas en este espacio protegido desde su declaración se ha primado en un alto grado las relacionadas con conservación frente a las vinculadas a los otros dos objetivos básicos asignados al Parque Natural andaluz, el desarrollo endógeno o el fomento del uso público.

El desarrollo de esta orientación predominante parece encontrar una justificación si se considera el elevado nivel de conservación general de los ecosistemas del Parque Natural, contexto en el que además destacan realidades muy relevantes desde un punto de vista natural tales como: la presencia de la única población de roble melojo (Quercus pyrenaica) conservada en la provincia de Córdoba, o la localización del que hoy se considera como el núcleo poblacional más importante de España (en continuidad con el del Parque Natural de la Sierra de Andujar) de lince ibérico (Lynx pardina).

Sin embargo, el desequilibrio apuntado alude, más allá de esta realidad evidente, a un hecho de mucha mayor profundidad y trascendencia en cuanto a la gestión de los Parques Naturales andaluces hasta el momento presente. En este sentido, la carencia de unos presupuestos y unas líneas de actuación específicas para los Parques de esta región ha venido determinando, en gran medida, el que las acciones desarrolladas en los mismos no hayan respondido de manera directa y concreta a las demandas de cada espacio ante los ambiciosos objetivos asignados a esta figura protectora. A ello han contribuido, igualmente, tanto la propia extensión de la red de Parques Naturales ${ }^{12}$, como el que su gestión haya recaído de manera exclusiva en la Administración ambiental autonómica. 
Es por todo ello por lo que la política seguida hasta la fecha -aunque no explicitada- por parte de dicha Administración ambiental ha sido la de fomentar el desarrollo de las actividades no relacionadas directamente con la conservación de los valores naturales (como el desarrollo endógeno o el uso público) sobre todo en aquellos espacios de condiciones más favorables para las mismas o en los que aquéllas se encontrasen en alguna medida consolidadas. Ello no constituye el caso, tal como podrá comprobarse a lo largo de las páginas siguientes y deducirse de lo señalado hasta este punto, del Parque Natural de la Sierra de Cardeña y Montoro.

Tomando como punto de partida el conjunto de factores explicativos aludidos (de indole territorial y aquellos más específicos concernientes a los instrumentos y medios para el manejo de este Parque Natural), a continuación profundizaremos en el análisis de las dimensiones fundamentales de la gestión de este espacio protegido. Ello se llevará a cabo a través de la consideración de una serie de aspectos que estimamos claves para la comprensión del verdadero alcance de la misma en el momento presente y sus perspectivas cara a un futuro.

\section{Pinares y consorcios: claves de una situación compleja}

Los espacios de repoblación forestal localizados dentro del perímetro protegido coinciden, en su práctica totalidad ${ }^{13}$, con montes consorciados con particulares por parte del Patrimonio Forestal del Estado desde los años cuarenta del siglo pasado (cuadro 3).

La cuestión de los montes consorciados y convenidos en el Parque Natural de la Sierra de Cardeña y Montoro no cabe calificarla sino como compleja. Tal complejidad posee su fundamento, esencialmente, en el estado de la mayor parte de los montes que se encuentran bajo dicha situación jurídica. Y es que las masas de pinar locali-

Cuadro 3. Consorcios y convenios en el Parque Natural Sierra de Cardeña y Montoro (año 2003).

\begin{tabular}{|lccc|}
\hline & Número & Superficie (has) & $\begin{array}{c}\text { Número de montes } \\
\text { no repoblados }\end{array}$ \\
\hline Montes consorciados y convenidos & 19 & $7.948^{\prime} 32$ & 3 \\
Montes desconsorciados & 2 & $1.463^{\prime} 50$ & - \\
Montes en desconsorcio & 2 & $1.652^{\prime} 60$ & - \\
\hline
\end{tabular}

Fuente: Elaboración propia a partir del SIGMA, Bases de los Consorcios, y cartografía del Parque Natural.

* El único convenio del Parque Natural corresponde a una porción de 863 hectáreas del monte de propiedad particular denominado "Santa María". 
zadas en los mismos presentan, como característica general, unas densidades excesivas, debido al hecho de no haber sido objeto de tratamientos adecuados a sus condiciones desde el momento de su plantación hasta prácticamente los años noventa, lo cual constituye, obviamente, una fuente potencial de peligro en diversos aspectos (debilitamiento de la masa, mayor exposición de la misma a plagas forestales, elevado riesgo de incendios...).

Fue a la luz de esta realidad que se planteó, desde los primeros años de existencia del Parque Natural, por parte de la Administración ambiental andaluza, la necesidad de emprender las labores de saneo y mejora de la estructura física de estas masas de pinar. Ello se ha venido llevando a cabo mediante una serie de tratamientos selvícolas, entre los que el clareo de las masas de Pinus pinea y Pinus pinaster ha resultado la labor más destacada desde el punto de vista de su repercusión territorial. Tales claras han facilitado, además de la disminución de las densidades previamente existentes, tanto el crecimiento de los pies de pinar que se han respetado (el cual se ha visto fomentado, asimismo, por las podas de realce realizadas sobre dichos pies), como la regeneración del arbolado autóctono de quercíneas (fundamentalmente de encinas, alcornoques y quejigos). La recuperación de dicho arbolado autóctono, contemplada como norma a seguir en el artículo 45 del PRUG del Parque Natural, se lleva a cabo mediante la paulatina puesta en luz de los pies existentes de quercíneas, en los que se vienen llevando a cabo, además, podas de guiado y formación.

Las superficies afectadas por estos trabajos (cuadro 4), muestran que se ha venido realizando un muy notable esfuerzo en esta dirección, pese a las dificultades existentes y a las que seguidamente se hará referencia.

De hecho, a pesar de las considerables superficies de pinar trabajadas durante los últimos años, éstas no representan aún ni la tercera parte de las más de 8.000 hectáreas de coníferas localizadas dentro del área protegida, y aún no se ha trabajado de segundas en ningún cantón. La línea seguida a este respecto ha sido la de tratar de atender a las prioridades que resulten más apremiantes en función de lo contemplado en los Proyectos de Ordenación de montes, si bien las acusadas limitaciones presupuestarias existentes impiden en muchos casos trabajar en profundidad en todos los montes.

Pero, más que en las superficies afectadas, el esfuerzo que la ejecución de estos trabajos conlleva para la Administración se manifiesta sobre todo a través de las inversiones dedicadas a los mismos, que suponen un porcentaje elevadísimo (56 $51 \%)$ de los gastos totales realizados en este Parque Natural (cuadro 1). Resulta obvio que la motivación principal de la Administración con respecto a estas superficies forestales es la mejora de sus condiciones ambientales. Esta tarea se ha venido planteado como absolutamente ineludible, y ello por dos razones fundamentales: a tenor, primera- 
Cuadro 4. Superficie (en has/pies) afectada por tratamientos selvícolas en montes consorciados y convenidos del Parque Natural Sierra de Cardeña y Montoro (1991-2002)*.

\begin{tabular}{|lccc|}
\hline Año & Clareo y poda & $\begin{array}{c}\text { Poda para realce } \\
\text { del pinar }\end{array}$ & $\begin{array}{c}\text { Poda de guiado y } \\
\text { formación de quercineas }\end{array}$ \\
\hline 1991 & 126 & $216.000^{* * *}$ & $1.600^{* *}$ \\
1992 & 95 & $290.250^{* *}$ & $800^{* *}$ \\
1993 & $127^{\prime} 5$ & - & - \\
1994 & $199^{\prime} 8$ & - & $1.400^{* *}$ \\
1995 & $608^{\prime *} 7$ & - & $5.000^{* *}$ \\
1996 & 166 & $48^{\prime} 5$ & $7.272^{* *}$ \\
1997 & 263 & $50^{\prime} 5$ & 15 \\
1998 & 112 & - & 41 \\
1999 & $39^{\prime} 3$ & $101^{\prime} 5$ & - \\
2000 & $298^{*} 34$ & - & - \\
2001 & $406^{*} 76$ & 150 & 55 \\
2002 & - & $45.057^{* *}$ & - \\
Total & $2.442^{*} 4$ & - & - \\
\hline
\end{tabular}

Fuente: Elaboración propia a partir de las Memorias Anuales de Actividades del Parque Natural de la Sierra de Cardeña y Montoro.

* Datos referidos tanto a los montes consorciados y convenidos como al monte público "La Vegueta".

** Número de pies (al recoger las Memorias los datos sobre podas algunos años por hectáreas afectadas y otros por número de pies podados no resulta posible unificar las cifras para ofrecer datos globales).

mente, del estado lamentable en que se encontraban muchas de estas masas, las cuales se vieron incluidas a partir de 1989 dentro de los límites de un espacio protegido, al encontrarse insertas en un contexto más amplio de notable calidad ambiental; y, en segundo lugar, por la propia responsabilidad que con respecto a las mismas incumbe a la Administración, al ser la propietaria del vuelo en los consorcios establecidos.

Sin embargo, no resulta menos evidente el que la gestión de estas masas se ha convertido en un verdadero lastre para los responsables de la Administración ambiental, ya que al alto coste de las tareas necesarias en estos montes hay que añadir la escasa rentabilidad -en términos económicos- de los mismos. Este hecho se explica a partir del escaso porte, en general, de las masas presentes en el Parque (por la ausencia de tratamientos selvícolas previos, o la inadaptación de unas especies de coníferas que en este ámbito se encuentran fuera de su óptimo ecológico), pero, sobre todo, del gran descenso que se ha venido registrando en el precio de la madera ${ }^{14}$.

Habrá que esperar aún unos años para observar la evolución de los montes consorciados en el Parque Natural. En este punto sólo pretendíamos mostrar la comple- 
jidad de un problema que, sin constituir una de las cuestiones vinculadas más específicamente a la gestión de los espacios protegidos, absorbe buena parte de los ya de por sí escasos recursos destinados a la gestión de este Parque Natural.

\section{Una propiedad pública marcada por su insignificancia y su alto grado de protección}

La realidad de la propiedad pública en el Parque Natural de la Sierra de Cardeña y Montoro puede calificarse como de testimonial, ya que al margen de $4^{\prime} 12$ hectáreas propiedad del Ayuntamiento de Cardeña en el enclave de Aldea Cerezo, existe tan sólo un monte público (propiedad de la Junta de Andalucía) dentro del perímetro protegido: el denominado "La Vegueta", de 526377 hectáreas, formado por la agrupación de tres antiguos montes de propiedad estatal ${ }^{15}$, y localizado en un enclave situado entre el núcleo de Aldea Cerezo y el Río Yeguas, curso fluvial que marca el límite oriental de este espacio protegido separándolo del Parque Natural de la Sierra de Andujar (Jaén).

Este enclave se considera como Área de Alto Interés Ecológico en la zonificación establecida por el PORN del Parque, es decir, como una de las zonas de máxima protección (Grado A) del mismo. De hecho, según lo establecido en la regulación recogida en dicho instrumento de planificación, "las actividades preferentes a realizar en estas zonas estarán relacionadas con la conservación, investigación e interpretación de la naturaleza", requiriéndose en todo caso autorización de la Administración ambiental tanto para el acceso a las mismas como para "los usos y aprovechamientos a realizar", los cuales, además, "estarán subordinados a los fines de conservación de sus valores naturales" (Consejería de Medio Ambiente, 1996b, 63). En cuanto al por qué de la dotación de un alto grado de protección a este enclave, no cabe duda que éste posee su fundamento, en buena medida, en los valores intrínsecos a dicho ámbito: carácter limítrofe con un área de una riqueza faunística excepcional como el Valle del Yeguas, presencia de algunas masas de bosque mediterráneo autóctono en gran estado de conservación, destacadas formaciones de enebrales,... Sin embargo, no se puede obviar el hecho de que el factor decisivo que ha contribuido a la dotación de este rígido régimen de protección reside en la propia condición de dominio público de este ámbito. Ello se prueba al constatar el hecho de que la relevancia de los valores naturales del mismo, aún siendo muy notable, no es mayor a la existente en otras porciones de este espacio protegido, las cuales, sin embargo, al encontrarse en manos privadas, gozan de un régimen de protección menor (Grado B). Esta realidad, referente al establecimiento de la zonificación en muchos espacios protegidos no tanto en función de una racionalidad ecológica como del tipo de propiedad exis- 
tente (en un intento de evitar roces con los titulares de predios privados), ha sido recientemente puesta de manifiesto por Mulero Mendigorri (2002), el cual advierte del riesgo que ello supone para la salvaguarda de los propios valores naturales presentes en el espacio.

La propiedad pública ha sido objeto, desde la creación del Parque, de varias actuaciones y proyectos. Entre estas actuaciones, que han tenido en todo caso una incidencia territorial bastante puntual y limitada, destacan tratamientos selvícolas en las masas de pinar existentes en el monte La Vegueta o labores diversas en base a Programas de Conservación de Especies Amenazadas de la Fauna andaluza (cuadro 4).

Cabe pensar que la escasa significación superficial de la propiedad pública no permite la implementación -por parte de la Administración- de una línea de actua-

Cuadro 4. Principales programas y acciones desarrollados en el monte público "La Vegueta" (1989-2002).

\begin{tabular}{|c|c|}
\hline Programas de actuación & Acciones concretas \\
\hline Tratamientos selvícolas en masas de pinar & $\begin{array}{l}\text { - Clareo y poda de pinares con densidades } \\
\text { excesivas } \\
\text { - Puesta en luz y poda de guiado y formación } \\
\text { de quercineas } \\
\text { - Aprovechamientos maderables } \\
\text { - Recogida y eliminación de residuos }\end{array}$ \\
\hline $\begin{array}{l}\text { Programa de lucha integrada contra la procesiona- } \\
\text { ria del pino }\end{array}$ & - Colocación de trampas de feromona \\
\hline $\begin{array}{l}\text { Programa de tratamientos contra los perforadores } \\
\text { del pino }\end{array}$ & - Tratamientos mecánicos (corta y quema) \\
\hline $\begin{array}{l}\text { Proyecto -cofinanciado con fonclos LIFE- de } \\
\text { Regeneración de Hábitats Críticos para la } \\
\text { Supervivencia del Águila Imperial. }\end{array}$ & $\begin{array}{l}\text { - Acondicionamiento del terreno y creación de } \\
\text { pastizales } \\
\text { - Construcción de vivares para repoblación de } \\
\text { conejos }\end{array}$ \\
\hline Adjudicación gratuita de asentamientos apícolas & $\begin{array}{l}\text { - Instalación de un aprovechamiento apicola en } \\
\text { cada uno de los tres montes que conforman la } \\
\text { finca "La Vegueta" }\end{array}$ \\
\hline $\begin{array}{l}\text { Proyecto para la conservación de la flora en la } \\
\text { provincia de Córdoba }\end{array}$ & - Plantaciones de almez \\
\hline $\begin{array}{l}\text { Programa de actuaciones para la conservación de } \\
\text { aves carroñeras en Andalucía }\end{array}$ & - Construcción de un muladar \\
\hline $\begin{array}{l}\text { Programa de actuaciones para la conservación del } \\
\text { lince en Andalucía }\end{array}$ & $\begin{array}{l}\text { - Construcción de cercados para cría de conejo } \\
\text { e instalación de majanos artificiales } \\
\text { - Realización de desbroces manuales, siembra } \\
\text { de avena y veza, y abonado de pracleras }\end{array}$ \\
\hline
\end{tabular}

Fuente: Elaboración propia a partir de las Memorias Anuales de Actividades del Parque Natural. 
ción estable y variada en cuanto a los proyectos y acciones que pudiese contemplar, tanto desde la óptica de la conservación como del fomento del uso público en este espacio protegido. Ello, sin embargo, tal como se analizará a continuación, si bien es cierto para la cuestión del uso público, no parece serlo tanto para la conservación y el fomento de los valores naturales de este espacio, ya que el papel de los predios de particulares se contempla cada vez más a ese respecto como fundamental.

\section{La implicación administrativa respecto a las fincas privadas del Parque Natural: el deseo de aunar conservación y desarrollo}

Los predios de propiedad particular gozan de una gran relevancia territorial en el Parque Natural ya que, como se sabe, suponen el $77^{\prime} 96 \%$ de su superficie, a lo que hay que añadir el 20,66\% que engloban los terrenos privados en régimen de consorcio (cuadro 1).

Desde la óptica de la Administración se contemplan, hoy día, estos terrenos en manos privadas como elementos claves tanto para la conservación y mejora de los valores naturales de este espacio protegido, como para la dinamización socioeconómica de la comarca en que éste se asienta. A continuación -en consonancia con el planteamiento adoptado para este trabajo- realizaremos una breve síntesis y valoración de las principales líneas de actuación administrativa con respecto a la propiedad privada del Parque a la luz de los objetivos anteriormente referidos.

Hay que recordar que en la zonificación del Parque Natural, la gran mayoría de los terrenos en manos de particulares son catalogados como zonas de Grado B, con un régimen de protección no tan restrictivo como en los enclaves de Grado A. Según el artículo 151.1 del PORN, "las actividades preferentes a realizar en estas zonas han de estar relacionadas con la conservación de la naturaleza, así como con el desarrollo de actividades productivas primarias compatibles con la estabilidad de estos ecosistemas" (Consejería de Medio Ambiente, 1996b, 64). Las normas específicas establecidas en virtud de dicha zonificación (artículo 151.2 del PORN) no suponen unas cortapisas especialmente limitantes, a excepción de algunas restricciones en cuanto a cambio de usos del suelo, construcciones y a otras actividades especialmente impactantes tales como la apertura de nuevos caminos y canteras ${ }^{16}$ (Consejería de Medio Ambiente, 1996b). En relación con la relativa laxitud de este régimen de protección con respecto sobre todo a las actividades agrarias, cabe recordar el muy notable peso de que gozan tales actividades en la práctica totalidad de las fincas de particulares. 
La extensividad de la mayor parte de los aprovechamientos constituye en ese sentido un factor explicativo esencial del buen estado de conservación general patente en muchos de los predios de propiedad particular, el cual, se ve, a su vez, facilitado por la ejecución de diferentes labores sujetas a autorización administrativa. Dichas labores presentan, en general, una gran relevancia territorial en este espacio protegido (cuadro 5) ${ }^{17}$, y poseen, a su vez, muy distintas funciones: revitalizar los pies de quercíneas y favorecer la producción de bellotas (poda), eliminación del matorral serial para mantener o crear espacios de pastizal en la dehesa (desbroce), transformación de brotes arbustivos en pies arbóreos (resalveo), y dotación de un alimento adicional para las reses, tanto ganaderas como cinegéticas (siembra de gramíneas y leguminosas).

Cuadro 5. Superficies (en has $/ \mathrm{n}^{\circ}$ pies) autorizadas para el desarrollo de labores de conservación y manejo de masas forestales en fincas de particulares (1990-2002)*

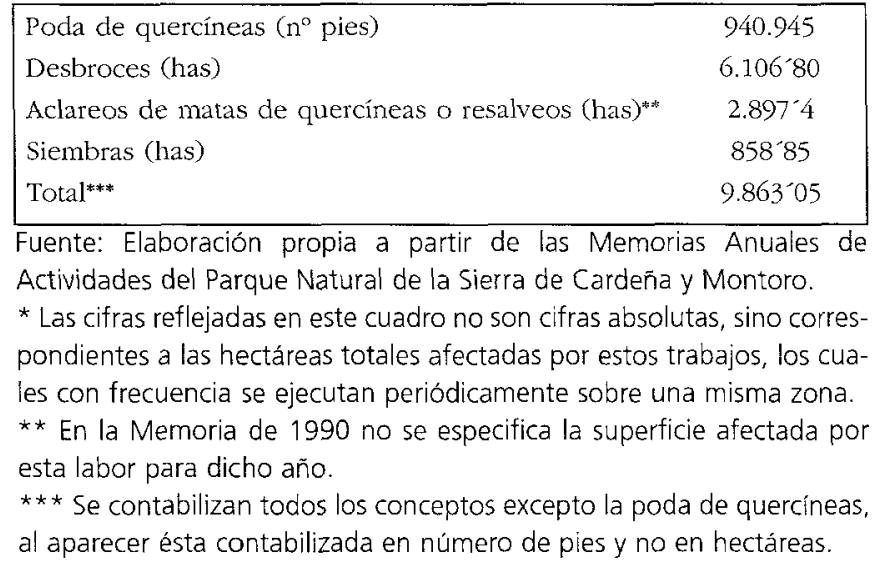

Resulta obvio el hecho de que tales trabajos se realizan, por parte del particular, con el objetivo fundamental de facilitar el aprovechamiento agrario (esencialmente ganadero y cinegético) de sus fincas; ello no es óbice, en todo caso, para la virtualidad de los mismos en el sentido de permitir e incluso fomentar la conservación y regeneración de la cubierta vegetal (sobre todo de los espacios adehesados) en caso de que se lleven a cabo adecuadamente. En este sentido, el papel de la Administración es el de velar por la correcta ejecución de los trabajos, en función de unas normas reguladoras que no son distintas de las existentes para terrenos no protegidos, al establecerse -en la Ley Forestal 2/1992, de 15 de junio- un régimen administrativo general y común para el conjunto de los terrenos forestales andaluces en manos de particulares $^{18}$. El principal matiz diferenciador viene dado por el desarrollo de un control más 
de cerca de las labores realizadas, al llevarse dicho control, para los terrenos situados dentro de los límites del Parque Natural, desde la Dirección del mismo.

Sin embargo, a pesar de esta importante labor administrativa y de control de actuaciones en terrenos del Parque Natural por parte de sus responsables administrativos, la paradoja surge al comprobar cómo éstos no gozan de un control real sobre algunos de los principales aprovechamientos que se llevan a cabo en el mismo ${ }^{19}$, en razón de la diversidad competencial y la falta de coordinación y colaboración interadministrativa existente a este respecto. Así por ejemplo, el control de los aprovechamientos agrícolas y ganaderos corresponde, también dentro de los límites del espacio protegido, a la Administración agraria andaluza -Consejería de Agricultura y Pesca-, la cual no vela en ocasiones, sin embargo, por el estricto cumplimiento de los criterios que para la ordenación de tales actividades se recogen en el PORN del Parque Natural (Mulero Mendigorri, 2001).

Como consecuencia de ello, son patentes, en la actualidad, algunas amenazas a este respecto, tales como los problemas de erosión del suelo en una cuarta parte de los cultivos de olivar, o la transformación de algunas explotaciones ganaderas extensivas en semiextensivas (Consejería de Medio Ambiente, 2001b), derivadas tanto de la concentración de cabezas de ganado en las fincas como de la creciente compatibilidad cinegético-ganadera en algunas de ellas. A pesar de ello, en la actualidad el principal problema para la adecuada conservación de la cubierta vegetal lo constituyen las cargas excesivas vinculadas a cotos de caza mayor (en función, sobre todo, de la superpoblación de hembras de ciervo), hecho agravado ante la gran expansión reciente de los cercados cinegéticos. Sin embargo, en este caso, sí se posee control efectivo -al menos en teoría- por parte de la Administración ambiental, el cual ha venido siendo ejercido mediante la implementación de una serie de medidas como la autorización de la caza selectiva y las batidas de gestión.

No obstante, pese a éstas y otras amenazas existentes (no respeto en algún caso de las condiciones de autorización para la realización de tareas forestales y construcciones, fundamentalmente) ${ }^{20}$, el estado de conservación del medio natural en este Parque Natural cabe calificarlo, en términos generales, como satisfactorio. Ello justifica la implementación por parte de la Administración ambiental andaluza de una nueva línea de acción: la firma de convenios con titulares de fincas para el desarrollo de programas de actuaciones -diseñados a nivel regional- para la conservación de especies de flora y fauna amenazadas aún presentes en este territorio. Esta línea, contemplada por los responsables de la gestión del Parque como mucho más rentable y eficaz que la adquisición de fincas, ha comenzado ya a aplicarse para el caso de algunas especies emblemáticas de este espacio: de este modo, en 2002 se firmaron 13 convenios de colaboración con otras tantas fincas del Parque Natural en el marco de los Programas de Actuaciones para la Conservación del Lince Ibérico y el Águila Imperial Ibérica. Las actuaciones que han comenzado a realizarse se destinan fundamentalmente a la recuperación de las poblaciones de conejo (presa básica para 
ambas especies) en amplios sectores del Parque Natural: cercados para cría, construcción de majanos artificiales, desbroces manuales, abonado de paraderas, y siembras de avena y veza (Consejería de Medio Ambiente, 2002).

Se espera, en relación con ello, que se pueda salvar una hipotética oposición de los propietarios sobre la base de las contrapartidas que éstos reciban (revitalización de las poblaciones de conejos -especie fundamental para la caza menor-, mejora del estado de conservación de determinadas parcelas de sus fincas...). Será necesario, en todo caso, esperar algunos años para calibrar la viabilidad así como las verdaderas repercusiones de estas iniciativas.

Pero, como igualmente se señalaba con anterioridad, desde la Administración se contempla este espacio protegido también como un posible factor de dinamización que pudiese canalizar un desarrollo sostenible para la zona. En torno a esta realidad, que ha resultado mucho menos potenciada -desde instancias administrativas- que la conservación ${ }^{21}$, se han despertado recientemente nuevas expectativas a partir del comienzo de la elaboración, en los primeros meses de $2003^{22}$, del Plan de Desarrollo Sostenible (PDS) ${ }^{23}$ para este Parque Natural. Cabe aclarar que el inicio de los trabajos para su elaboración se plantea en un contexto más global en el que este instrumento se encuentra en la actualidad ya operativo en otros diez Parques andaluces. El hecho de que el Parque Natural de la Sierra de Cardeña y Montoro se encuentre entre los últimos espacios para los que se ha acometido la elaboración de dicho instrumento planificador no puede disociarse, a nuestro juicio, de los condicionantes especialmente negativos que presenta este territorio para la aplicación de un desarrollo endógeno realmente operativo: su débil potencial demográfico, la muy escasa diversificación productiva, el absoluto predominio de las grandes propiedades en su perímetro protegido, etc...

Pese a ello, la implicación real en los primeros talleres de participación estratégica para su elaboración de todos los sectores productivos de la comarca así como del conjunto de instancias administrativas -no sólo la medioambiental- que de una u otra forma puedan tener una incidencia sobre el territorio afectado (Consejería de Medio Ambiente, 2002) parece augurar unas buenas perspectivas al respecto. Creemos que sólo partiendo de estas premisas podrá elaborarse un Plan que implique una auténtica gestión territorial, una gestión integral -no estrictamente conservacionista- pero sostenible, que permita aprovechar racionalmente las plusvalías ambientales de la zona.

\section{El limitado desarrollo del uso público}

Uno de los factores que ha venido contribuyendo más directamente a la revitalización socioeconómica y territorial de no pocos espacios protegidos andaluces ha sido 
el fomento del uso público (Bueno, 1997). En el caso del Parque Natural de la Sierra de Cardeña y Montoro, no cabe duda que existen grandes potencialidades en este sentido: buenas comunicaciones tanto con la capital cordobesa y el resto de poblaciones emplazadas en el Valle del Guadalquivir como con la vecina comarca de Los Pedroches, gran calidad paisajistica, e importantes valores florísticos y faunísticos ${ }^{24}$.

Sin embargo, la presencia testimonial de la propiedad pública en el Parque Natural así como la ausencia de adquisiciones desde su declaración sí suponen un importante escollo en este caso. Este problema, que podía pensarse en un principio como coyuntural, deviene en estructural cuando se toma en consideración la carencia de una política patrimonial propia para los espacios protegidos andaluces. Ello se ve agravado, a su vez, por las limitaciones presupuestarias existentes y, sobre todo, por la ausencia de una línea de actuación clara y definida al respecto de la adquisición de fincas por parte de la Administración ambiental. El reflejo de esto último lo encontramos en las numerosas notificaciones de tanteo y retracto (un total de cincuenta y seis) recogidas en las Memorias Anuales de Actividades para los años 1991-2002. Sin embargo, sólo en uno de esos casos (en el año 2002) la Junta de Andalucía se ha decidido a ejercer el derecho que le otorga el artículo 24 de la Ley autonómica 2/1989, a pesar de que han sido varios los informes favorables emitidos por los gestores del Parque Natural, conscientes de la necesidad de ampliar el patrimonio público de este espacio protegido con el fin de posibilitar una gestión más adecuada del uso público.

Y es que el uso público en el Parque Natural de la Sierra de Cardeña y Montoro ha estado marcado por una serie de limitaciones muy nítidas entre las que destacan sobremanera las escasas inversiones aplicadas (cuadro 2), traducidas en unas grandes carencias a nivel de infraestructuras: escasez de la red de senderos -cinco-, caracterizada además por su poca representatividad al transcurrir todos ellos de manera casi exclusiva a través de ecosistemas de dehesa; pero, sobre todo, la ausencia de algunos equipamientos absolutamente básicos y presentes desde años atrás en la gran mayoría de Parques Naturales andaluces (Consejería de Medio Ambiente, 2002b), tales como las áreas recreativas o el Centro de Recepción e Interpretación, el cual ha sido objeto de continuos retrasos en cuanto a su ejecución y que no ha entrado en funcionamiento hasta los primeros meses de $2004^{25}$. Sin duda, la situación de este espacio a nivel de dotación de equipamientos para el uso público por parte de la Administración ambiental andaluza lo sitúa a la cola de los Parques de la región.

Junto a todo ello, la regulación del uso recreativo en este espacio se ve afectada igualmente por la inexistencia de instrumentos que permitan una planificación adecuada del mismo en el Parque Natural. De ello es buena muestra la carencia, por el momento, del Programa de Uso Público, instrumento planificador concebido como herramienta de desarrollo del PRUG (Consejería de Medio Ambiente, 1996b, 77) para el diseño de un modelo definido de uso público para este Parque Natural y con el que ya cuentan seis Parques Naturales andaluces (www.juntadeandalucia.es/medioambiente). La ausencia de 
dicho modelo y de una ordenación efectiva de esta actividad se traduce generalmente en ejemplos patentes de escasa calidad en el servicio de uso público de los Parques Naturales (Gordi Serrat, 1994); éste es el caso de la Sierra de Cardeña y Montoro, donde se ha venido autorizando la realización de acampadas en los alrededores de Aldea Cerezo, en una zona de titularidad del Ayuntamiento de Cardeña no habilitada específicamente para ello, pero utilizada como tal ante la inexistencia de zonas específicas de acampada.

En el momento presente parecen adivinarse nuevas y algo más alentadoras perspectivas para el uso público, tales como la ya referida apertura del Centro de Visitantes, la creación de una zona de acampada en la zona de dehesa emplazada entre Cardeña y El Cerezo (en caso de que finalmente se consiga la adquisición de la finca para la que se ha decidido a ejercer el derecho de retracto), o la ampliación de la red de senderos actualmente existente.

Ahora bien, más allá de este tipo iniciativas aisladas ${ }^{26}$, sería más conveniente la definición de un modelo definido, coherente y unitario en esta cuestión, que permita el desarrollo de un uso público ordenado, respetuoso con la fragilidad del entorno, pero que, al mismo tiempo, sea un uso público de calidad y que permita entrar en contacto con una porción suficientemente representativa y variada de los valores naturales y culturales de este territorio. Éste es, sin duda, el gran reto que en esta cuestión se plantea en la actualidad. El Programa de Uso Público (actualmente en elaboración) puede constituirse en el instrumento adecuado que permita la definición de dicho modelo de uso público adaptado a la especificidad de este espacio.

\section{Reflexiones finales: la necesidad de una gestión territorial equilibrada}

Se ha venido apuntando, a lo largo de este trabajo, la existencia de un notable desequilibrio entre los Parques Naturales andaluces con respecto a la consecución de los tres objetivos comunes atribuidos a los mismos, con especial incidencia en el desarrollo endógeno sostenible y el impulso del uso público recreativo.

Uno de los espacios más desfavorecidos a este respecto ha sido el Parque Natural de la Sierra de Cardeña y Montoro, cuya gestión administrativa ha resultado ser, hasta la fecha, bastante desigual, al primarse muy preferentemente los objetivos de conservación sobre los restantes objetivos planteados desde un principio.

Las potencialidades de este territorio en relación con ellos (buena accesibilidad, destacados valores naturales, culturales y paisajísticos, actividades agrarias consolidadas...) han sido esbozadas a lo largo de este trabajo, al igual que las propias limita- 
ciones existentes (predominio de la propiedad privada, debilidad y escaso dinamismo del elemento poblacional...). Resulta evidente, en todo caso, que en el momento actual comienzan a darse algunos pasos en este sentido, los cuales, sin embargo, no deberían ser planteados, en nuestra opinión, a modo de iniciativas inconexas y voluntaristas, sino como parte de una estrategia general de gestión territorial equilibrada y adaptada a la especificidad de esta subcomarca del noreste cordobés.

La definición en el nuevo PRUG del Parque Natural de unas propuestas de actuaciones estimadas como prioritarias en función de las necesidades detectadas en este espacio protegido puede interpretarse como un primer logro en esa línea. De hecho, la lectura de dichas prioridades de actuación alude directamente a muchas de las cuestiones y retos esbozados a través de las páginas anteriores. Podemos destacar, a modo ilustrativo, los siguientes aspectos:

- "Realización de tareas de repoblación en aquellas zonas en las que no se asegure la regeneración natural (..)

- Realización de estudios de carga ganadera en las fincas del Parque Natural dedicadas a estos aprovechamientos.

- Incentivo de las razas ganaderas autóctonas así como de la ganadería ecológica (..)

- Dentro de la ampliación general recomendable de la oferta de uso público, será necesaria la instalación de un complejo de uso público y educación ambiental (..)

- Aumentar la oferta de puntos de información sobre el Parque Natural" (Consejería de Medio Ambiente, 2003).

Estos ejemplos parecen atisbar el deseo de definir, por vez primera, un modelo realmente consolidado y global de acción para este espacio protegido, que no excluya ninguna de las grandes directrices de acción a las que debe aspirar en cuanto que Parque Natural.

Ahora bien, estimamos que el éxito de dicho modelo en la práctica depende en gran medida de la consecución de dos requisitos básicos a ese respecto. El primero de ellos se refiere a la necesidad de concretar tales prioridades de actuación en verdaderos programas operativos, que se diseñasen en el marco de un presupuesto específico; la concreción de dichas acciones debería recaer, en todo caso, en la Dirección del Parque Natural ${ }^{27}$, con lo cual se aseguraría, a nuestro entender, una mejor adaptación a la propia realidad y demandas del Parque. Junto a ello, el segundo requisito, complementario del anterior, alude a la posibilidad de implicar más activamente en la gestión cotidiana del espacio a aquellas otras instancias administrativas (fundamentalmente agrícolas y turísticas, así como entidades locales) con competencias directas con respecto a la conservación y fomento de los recursos específicos presentes en el mismo. 
Estas directrices, que definen en sus líneas básicas lo que desde fechas recientes se ha venido catalogando bajo el concepto de gestión activa (Gómez-Limón et al., 2000), podrían coadyuvar, indudablemente, a la superación de algunas de las lagunas y disparidades patentes en la gestión territorial y ambiental de espacios como el Parque Natural de la Sierra de Cardeña y Montoro. De este modo, las expectativas depositadas en la protección como medio para la conservación y revalorización de áreas rurales podrán comenzar a encontrar una respuesta más adecuada y equilibrada.

\section{Notas}

1 Los primeros Parques Naturales en Andalucía fueron declarados a partir de 1984 (fecha del traspaso de competencias en materia de medioambiente a la Comunidad Autónoma andaluza), si bien su plasmación legal definitiva en el contexto andaluz se produjo con la aparición de la Ley $2 / 1989$, de 18 de julio, por el que se aprueba el inventario de espacios naturales protegidos de Andalucía y se establecen mediclas adicionales para su protección.

2 Quiero agradecer, muy sinceramente, a José Manuel Quero, Director-Conservador del Parque Natural de la Sierra de Cardeña y Montoro, la amable colaboración prestada en todo momento.

3 Ésta es la superficie real del Parque Natural (cuyos límites fueron precisados en 1998 sobre la Ortofotografía del SIG Oleícola del Ministerio de Agricultura y Pesca) y la contemplada en la revisión del PORN y PRUG del Parque Natural, si bien la superficie que se ha venido atribuyendo a este espacio protegido hasta fechas muy recientes era de 41.212 hectáreas, por cuanto ésta última es la que aparece estipulada en la Ley de 1989 en virtud de la cual se declaraba este espacio protegido.

$4 \mathrm{El}$ núcleo de El Cerezo, si bien se encuentra igualmente situado dentro del Parque Natural (figura 1), no goza hoy día de población estable, siendo su único uso el de alojamiento de turismo rural (véase a ese respecto el apartado 5 de este mismo trabajo).

5 El más poblado de todo el cuadrante nororiental de la provincia cordobesa, con 9.509 habitantes en 2003 (www.juntadeandalucia.es/institutodetadistica/sima).

6 El total del municipio de Cardeña contaba en 2003 con 1.755 habitantes, y una muy débil densidad de población de $3,42 \mathrm{hab} / \mathrm{km}^{2}$ (www.juntadeandalucia.es/institutodeestadistica/sima).

7 Estas afirmaciones se fundamentan en el análisis del listado y mapa de cotos del Parque Natural y en la certeza de que la gran mayoría de los cotos de caza mayor son unipropiedad, aunque no es posible ofrecer datos más precisos ante la inexistencia de un estudio sobre la propiedad en el Parque.

8 Cabe reseñar, a modo ilustrativo de tal expansión, algunos datos relativos a la caza mayor en el Parque Natural durante la temporada cinegética 2001-2002: se celebraron 40 jornadas cinegéticas (bajo distintas modalidades, entre las que destacaron la de montería y montería mixta), con 1611 puestos, y un total de 2309 piezas cazadas (fundamentalmente ciervo y jabalí, y -en mucha menor medida- muflones y gamos).

9 La principal modificación en la zonificación establecida en el documento de renovación del PORN se refiere, precisamente, a los enclaves de olivar marginal, que han pasado a ser incluidos en las zonas de protección de Grado C. 
10 Rémora, ésta, que ha tratado de ser subsanada en el nuevo PRUG de 2003, en el que se contempla una propuesta de actuaciones consideradas como prioritarias en el Parque Natural durante el periodo de vigencia de dicho instrumento de planificación.

11 Que es el organismo encargado de manera exclusiva de la gestión de los Parques Naturales andaluces. 12 Conformada en la actualidad por 24 espacios, que abarcan un total de 1.416 .837 hectáreas (www.juntadeandalucia.es/medioambiente).

13 A excepción de una parte de la finca pública "La Vegueta" (véase próximo apartado).

14 Este descenso ha llegado a tal punto, que en la actualidad el valor de la misma es generalmente más bajo que el coste de las labores requeridas para obtenerla (corta, tronza, desembosque, apilado y eliminación de residuos). Por ello, desde hace unos años, muchos de los concursos ofertados a empresas para el aprovechamiento de la madera en montes del Parque Natural han quedado desiertos, requiriéndose la elaboración de proyectos diseñados por la Administración para la ejecución de los aprovechamientos.

15 Barranco Cerezo, Vega de Cantareras y Vegueta del Fresno.

16 Así, entre las regulaciones más significativas a este respecto destacan: la prohibición de las talas de árboles que conlleven una transformación del uso forestal del suelo, la imposibilidad de construir viviendas familiares aisladas si no se encuentran vinculadas directamente a las explotaciones agropecuarias, o la limitación a la construcción de nuevos accesos (salvo aquellos considerados imprescindibles para la gestión y el uso público, o el desarrollo de las actividades agrarias tradicionales).

17 En el cuadro 6 sólo aparecen contempladas aquellas actividades forestales más habituales o relevantes desde un punto de vista territorial en el Parque Natural, si bien no hay que obviar otras tareas más o menos puntuales como la corta de árboles secos, talas o la extracción de corcho (ciertamente escasa en este espacio por la presencia tan sólo de pequeñas manchas de alcornocal en el mismo).

18 Régimen que fue asumido en todos sus términos en el PORN del Parque Natural (artículos 49 a 63 ).

19 Aprovechamientos en virtud de los cuales -sobre todo en el caso de la explotación ganadera y cinegética- se llevan a cabo dichas labores forestales.

20 Una relación bastante detallada de los principales incumplimientos en el Parque Natural con respecto a la normativa aplicable en el mismo puede encontrarse en las Memorias Anuales de Actividades, en el apartado de "Expedientes sancionadores".

21 La única iniciativa que ha fructificado hasta la fecha en ese sentido ha sido la Marca Parque Natural de Andalucía, etiqueta creada como forma de revalorización de determinadas producciones y servicios locales de calidad y respetuosos con el medio ambiente en los Parques Naturales andaluces. Sin embargo, ésta se ha concedido tan solo a los alojamientos rurales habilitados en la antigua pedanía de El Cerezo, y a la miel producida en la empresa Apícola Montoro (www.juntadeandalucia.es/medioambiente).

22 Acuerdo de 10 de diciembre de 2002, del Consejo de Gobierno, por el que se aprueba la formulación del Plan de Desarrollo Sostenible del Parque Natural de la Sierra de Cardeña y Montoro (BOJA, $\mathrm{n}^{\circ} 3$, de 7 de enero de 2003).

23 El PDS es el instrumento de planificación creado por la Ley andaluza de espacios protegidos (Ley 2/89) con el objetivo de dinamizar la estructura socioeconómica de los territorios sometidos a protección bajo la figura de Parque Natural.

$24 \mathrm{Si}$ bien estos dos últimos factores constituyen, indudablemente, al mismo tiempo una importante limitación para un uso masivo de este Parque Natural, determinando la necesidad, tal como se incidirá más adelante, de regular y planificar dicha actividad en puntos concretos de este espacio. 
25 Una de las consecuencias más negativas de esta gran demora apuntada es el escaso rigor y fiabilidad de las estimaciones realizadas hasta la fecha acerca del número de visitantes y sus preferencias, las cuales podrían resultar de gran utilidad para una toma de conciencia de esta realidad y una correcta planificación de las actividades de uso público.

26 Que en todo caso no van a posibilitar más que la consecución de unos logros ya alcanzados con carácter general en la mayor parte de los Parques Naturales andaluces.

27 La cual es la encargada, según el artículo 11 del PRUG, de asumir "la responsabilidad ejecutiva de la administración y gestión del Parque" (Consejería de Medio Ambiente, 1996b).

\section{Bibliografía}

Azcárate y Bang, T. De (1986): Política de Parques Naturales en Andalucía, en Actas del I Congreso Nacional de Parques Naturales. Sevilla, Agencia de Medio Ambiente- Instituto de Promoción Industrial de Andalucía, p. 9-11.

Bueno, J. L. (1997): Oportunidades para el desarrollo local que ofrece el uso público en las áreas protegidas, en Ramírez Sanz, L. y Gómez-Limón, J. (eds.): Seminario sobre alternativas de desarrollo económico para la población local de los espacios naturales protegidos. Madrid, Fernando González Bernáldez-Sección del Estado Español de la Federación de Parques Naturales y Nacionales de Europa.

CONSEJERÍA DE MEDIO AMBIENTE (19902002): Memorias Anuales de Actividades del Parque Natural de la Sierra de Cardeña y Montoro (documento inédito).

CONSEJERÍA DE MEDIO AMBIENTE (1996b): Plan de Ordenación de los Recursos Naturales y Plan Rector de Uso y Gestión del Parque Natural de la Sierra de Cardeña y Montoro. Sevilla, Junta de Andalucía.

CONSEJERÍA DE MEDIO AMBIENTE (2001b): Caracterización y Diagnóstico del Parque
Natural de la Sierra de Cardeña y Montoro. Sevilla, Junta de Andalucía (documento inédito).

CONSEJERÍA DE MEDIO AMBIENTE (2002b): Red de espacios naturales protegidos de Andalucia: instalaciones y servicios. Sevilla, Junta de Andalucía (CD-Rom).

CONSEJERÍA DE MEDIO AMBIENTE (2003): Plan de Ordenación de los Recursos Naturales y Plan Rector de Uso y Gestión del Parque Natural de la Sierra de Cardeña y Montoro. HOJA $n^{\circ} 213$, de 5 de noviembre de 2003.

EUROPARC-ESPAÑA (2002): Plan de acción para los espacios naturales protegidos del Estado español. Madrid, Fundación Fernando González Bernáldez-Sección del Estado Español de la Federación de Parques Naturales y Nacionales de Europa.

Gómez-Limón, J. et al. (2000): Los espacios naturales protegidos del Estado Español en el umbral del siglo XXI: de la declaración a la gestión activa. Madrid, Fundación Fernando González Bernáldez.

Gómez Mendoza, J., Manuel Valdés, C., Sáez Pombo, E. (1994): La gestión territorial y 
ambiental de un parque metropolitano: el Parque Regional de la Cuenca Alta del Manzanares (Madrid), en Actas del VII Coloquio de Geografia Rural. Comunicaciones. Córdoba, AGE-Universidad de Córdoba.

Gordi Serrat, J. (1994): La planificación del uso público en los espacios naturales protegidos, en Actas del VII Coloquio de Geografia Rural. Comunicaciones. Córdoba, AGE-Universidad de Córdoba.
Mulero Mendigorri, A. (2001): Algunas limitaciones impuestas por la figura de Parque Natural al desarrollo agrario: el ejemplo de la Sierra de Hornachuelos (Córdoba), en Actas del XVII Congreso de Geógrafos Españoles. Oviedo, AGE-Universidad de Oviedo.

Mulero Mendigorri, A. (2002): La protección de espacios naturales en España. Antecedentes, contrastes territoriales, conflictos $y$ perspectivas. Madrid, Mundi-Prensa.

www.juntadeandalucia.es/institutodeestadistica/sima

www.juntadeandalucia.es/medioambiente 